\title{
Lateral resolution enhancement using programmable phase modulator in optical coherence tomography
}

\author{
Muhammad Faizan Shirazi ${ }^{\mathrm{a}}$, Nam Hyun Cho ${ }^{\mathrm{a}}$, Woonggyu Jung ${ }^{\mathrm{b}}$ and Jeehyun Kim ${ }^{\mathrm{a},{ }^{*}}$ \\ ${ }^{a}$ School of Electronics Engineering, Kyungpook National University, Daegu,702-701, Korea \\ ${ }^{b}$ School of Nano-Bioscience \& Chemical Engineering, Ulsan National Institute of Science and \\ Technology, Ulsan, Korea
}

\begin{abstract}
Adaptive optics plays an important role in the correction of high-order aberrations to enhance lateral resolution. An OCT system coupled with a programmable phase modulator is designed to verify lateral resolution improvement. The Hamamatsu high-resolution, non-pixelized, optically addressed light modulator (PAL-SLM) PPM X7550 series is used to correct aberrations, utilizing a simple method based on phase information from OCT images. The advantages of this method are its high simplicity and low cost. A raster scanning technique is adopted to scan samples covered with scattering suspension. Metal lines covered with scattering suspension are imaged, and a respective correction is applied to remove the aberration. Results demonstrate an improvement in lateral resolution on metal grid samples.
\end{abstract}

Keywords: OCT, lateral resolution, aberration correction, adaptive optics, PAL-SLM

\section{Introduction}

The human eye works as an optical system, the function of which is to focus an image on the retina. The cornea and crystalline lens are the important optical components of the eye; imperfections and misalignments in this optical system cause light to deviate from its original path, making the light unable to reach the fovea centralis via its desired path. As a result, the image formed at the retina becomes blurred and causes vision impairment, defined as optical aberrations [1,2]. Optical aberrations are categorized into lower order and higher order aberrations. Eyeglasses and contact lenses can correct lower order aberrations of defocus and astigmatism, but are unable to correct higher order aberrations, making it difficult to obtain high-resolution retinal imaging or to provide high-quality vision to patients suffering from higher order aberrations.

Optical coherence tomography (OCT) is a well-established technique used to image microstructures using the coherence property of light based on the interference phenomenon. The axial and transverse resolutions define the image quality, and are important to access information and diagnose any defect in a corresponding species of interest. Age-related macular degeneration (AMD), retinopathy and

\footnotetext{
*Address for correspondence: Jeehyun Kim, School of Electronics Engineering, Kyungpook National University, Daegu 702-701, Korea. Tel./Fax: 82-53-950-7221; E-mail: jeehk@knu.ac.kr.
}

0959-2989/15/\$35.00 @ 2015 - IOS Press and the authors. 
glaucoma retinal disease [3-6] can also be diagnosed with this technology. However, the retinal images obtained with OCT are of low quality due to aberrations in the optical system.

The axial resolution of the optical system is a function of the source, while lateral resolution can be adjusted by controlling the aberration with optical components in the sample path. Adaptive optics (AO) plays an important role in the correction of aberrations in the visual system at a microscopic lev$\mathrm{el}$, and demonstrates advantages in correcting aberrations in vision and ophthalmoscopes. Numerous techniques have been demonstrated for the correction of aberrations using different types of adaptive optics coupled with wavefront sensors in a feedback loop [7-9].

Adaptive optics was developed to minimize the aberration in the optical system, and has been implemented to overcome the effect of atmospheric turbulence in astronomical telescopes [10-12]. With this recent advancement, $\mathrm{AO}$ has also become extensively used in ophthalmology [13-15].

In this paper, a technique to improve lateral resolution of the sample using adaptive optics is described. The adaptive optics used here is accomplished with a programmable phase modulator (PPM) which acts as an aberration corrector in the optical path. The phase information is extracted from the interference pattern of an image without the use of a wavefront sensor. The compensated phase map is applied based on the transfer function of PPM. As a result, aberration is removed through the use of adaptive optics.

\section{OCT with adaptive optics}

A Michelson interferometer is employed to obtain the interference pattern of back-scattered light from the sample and a reference arm. A broadband light source is delivered through a single-mode optical fiber and launched into the system by a collimating lens (L1) as shown in Figure 1. The beam diameter is set to $1 \mathrm{~mm}$. Because the parallel aligned spatial light modulator (PAL-SLM) works on the principle of phase modulation with strong polarization dependence, after the reflection of incident light, the component along the liquid crystal molecule director is phase modulated. A polarizer (P) removes all other polarization states of light except the vertical state that is aligned parallel to the PAL-SLM. A beam splitter (BS1) divides light into sample and reference paths. Light transmitted through BS1 is defined as sample path light. When light enters the reference path, its path-length is modulated by a retro-reflector (RR) mounted on a voice coil. The voice coil is driven by a function generator which provides a sinusoidal wave with $10 \mathrm{~V}_{\mathrm{p} \text {-p }}$ (peak-to-peak voltage). The voltage can be modified to vary the depth of the A-scan. After passing the RR, reference path light reflects back from a mirror (M2) and returns to the BS1; by penetrating the BS1, the reference path light loses half of its optical power and reflects back from the mirror (M1) to recombine with the sample path light. No interference is observed at this position because path length difference between the reference and sample paths is longer than the coherence length of the source. Note that one-quarter of the reference path light can recombine with the sample path light, which can be minimized by using a polarizationsensitive beam splitter instead of using the BS1. The recombined light paths are then separated by the second-beam splitter (BS2). Half of the light transmitted through the BS2 enters the detection path, and is detected by a charge-coupled device (CCD) camera. Only one-eighth of the reference and sample path light can be detected by the CCD camera.

Sample path light reflected by the BS2 is expanded by a factor of ten using lenses L2 and L3, providing a $10 \mathrm{~mm}$ beam diameter incident on the adaptive optics (PAL-SLM). Because the size of the liquid crystal cell of the adaptive optics is fixed, magnification of the beam size can provide finer control over the optical field. The sample path light illuminates the reflective liquid crystal layer of the 


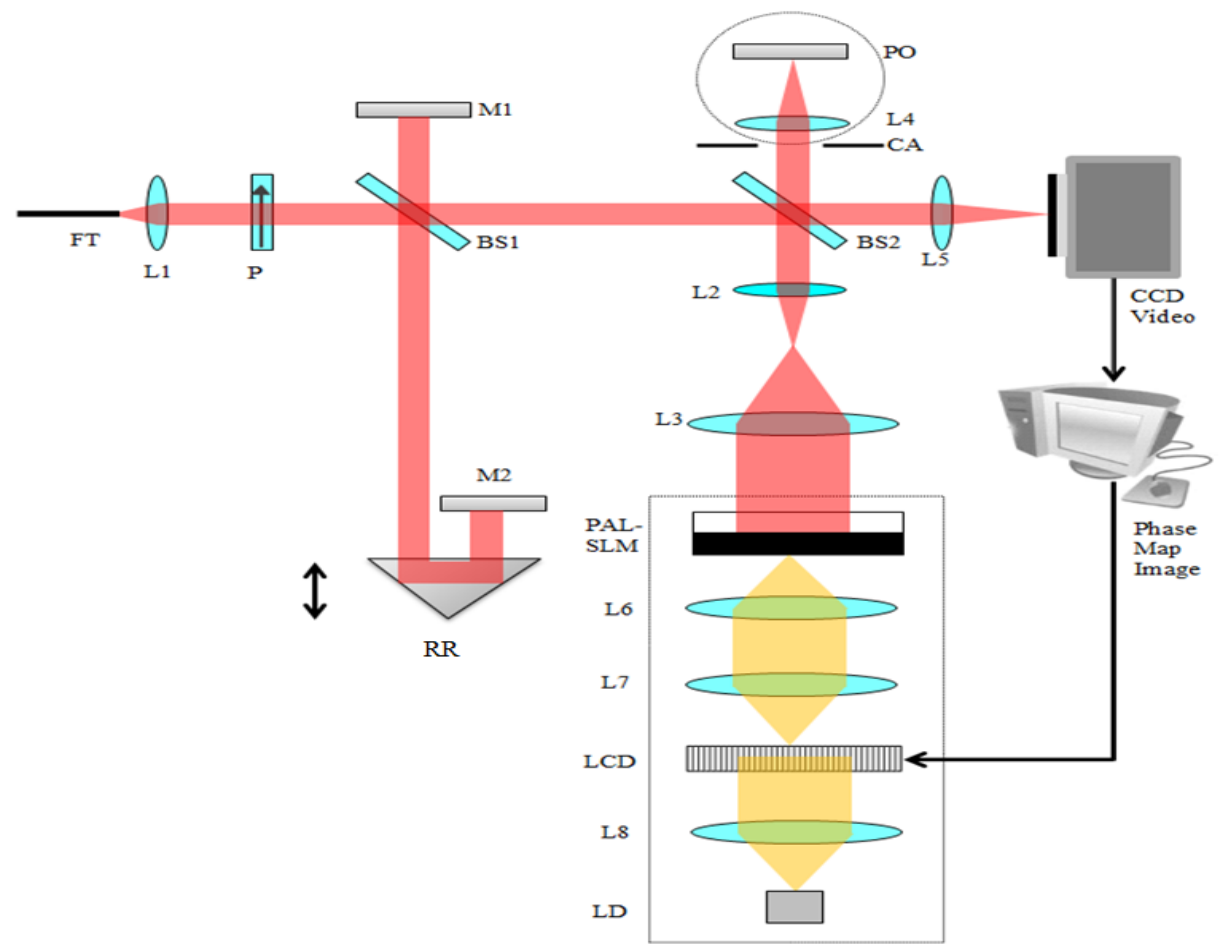

Fig. 1. Schematic diagram of parallel OCT system with adaptive optics. FT: optical fiber tip, LD: laser diode, RR: retroreflector, L: lens, LCD: liquid crystal display, PAL-SLM: parallel aligned spatial light modulator, BS: beam splitter.

PAL-SLM placed at the focal plane of lens L3 and undergoes wave-front modification. The phase information is extracted from the fringe pattern due to back-scattered light from the sample and reference paths. In turn, an intensity map is generated based on the transfer function of the PPM. This intensity map is inverted to compensate for wavefront due to aberrations. Hence, wavefront correction is achieved by applying this compensation intensity to the PPM. A detailed process of the wavefront modification has been articulated by previous research [16].

The modified sample path light returns to the BS2 and illuminates the sample. The liquid crystal layer is placed at a conjugate plane of the pupil plane (CA) by the relay lens pair (L2 and L3), so the adaptive optics compensate for the wavefront reflected back from the sample at this plane. A circular aperture (CA), a lens (L4), and a planar object (PO) construct a model eye. Incoming light is focused by L4 at the PO, which can be a scattering or specular reflector. This light uniformly illuminates the PO which is placed at the back focal plane of L4. The reflected light from the PO is imaged by lens pair L4 and L5 onto a CCD camera. An interference fringe forms at the detection plane of the CCD camera when the path lengths of the sample and reference paths are equivalent. The image of the PO captured by the CCD camera is blurred by the influence of the wavefront aberration of L4. However, this blurred image can be corrected by utilizing the phase information across the PAL-SLM, cancelling the wavefront aberration due to L4 so that the PAL-SLM performs as a wavefront corrector.

Figure 2(a) demonstrates the production of both $\mathrm{x}$ and $\mathrm{y}$ spatial frequencies by the diagonally-tilted aberration. The left half portion of this aberration is corrected using the compensating intensity map applied to the left half of the LCD of the PPM. After the aberration correction, the remaining half of the field displays fringe patterns as shown in Figure 2(b). 


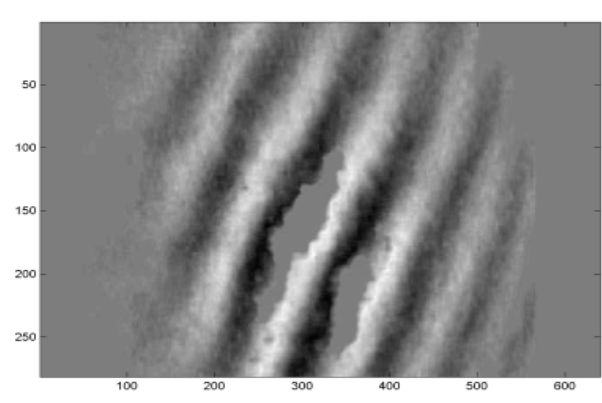

(a)

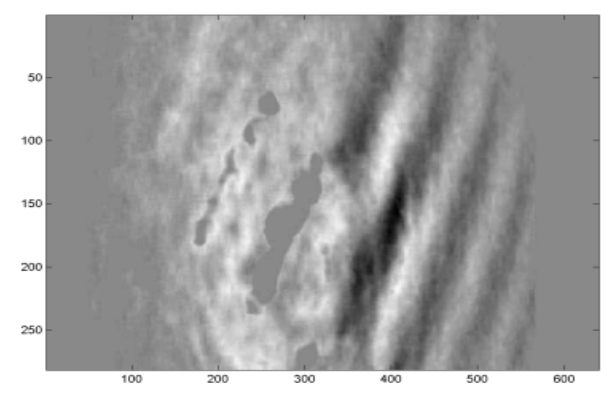

(b)

Fig. 2. (a) Interferogram of the diagonal tilt aberration; (b) compensated interferogram as correction applied to the left half of the field.

\section{Experimental setup}

Raster scanning OCT imaging was performed on the sample with adaptive optics to confirm improvement in lateral resolution. The sample consisted of a metal grid with several different metal lines on a glass substrate. Lines separated by $50 \mu \mathrm{m}$ were imaged.

Figure 3(a) shows the configuration of the sample stage. The metal grid was placed on a motorized stage which provided lateral scanning. The linear density of the metal lines of the image on the grid was 20 lines per millimeter. This metal grid had $25 \mu \mathrm{m}$ features that completely backscattered light, with an adjacent area made of glass. The separation between the metal lines was $50 \mu \mathrm{m}$, as shown in Figure 3(a).

The optical setup of the raster scanning OCT with adaptive optics is shown in Figure 3. For OCT imaging, the raster scanning method was used to increase the signal to noise ratio by using a photoreceiver in the detection path. The path included a flip mirror and a Si large-area photo-receiver $8 \mathrm{~mm}$ in diameter (Newfocus ${ }^{\mathrm{TM}}$, model 2031). When the flip mirror is folded down, the light passing through L5 was focused on the CCD camera. Once the CCD camera acquired the phase map containing the aberration information of the sample optics, the flip mirror was folded up to divert the light into the large area photo-receiver, and a lateral scan was performed.

A low-quality lens was used as an objective [L4] to introduce aberration. The focal length of the lens was approximately $18 \mathrm{~mm}$ with a beam diameter entering the lens of approximately $2 \mathrm{~mm}$, which yielded $9.7 \mu \mathrm{m}$ of calculated diffraction limited beam spot size at the sample surface.

A mode-locked Ti:Sapphire laser was coupled into a single-mode optical fiber and launched into the system through L1 with a power of $300 \mathrm{~mW}$. Light with $37 \mathrm{~mW}$ of power entered the sample path including the CA, L4, and PO. A variable neutral density filter was inserted between M1 and M2, and was adjusted to demonstrate comparable sample and reference powers at the detection plane.

To image the grid through scattered media, a cuvette with $300 \mu \mathrm{m}$ depth was covered with a metal grid, and filled with a suspension of $1 \mu \mathrm{m}$ micro-beads. Accurate density of the suspension was not traced, but suspensions were created with two different arbitrary densities. The scattering coefficient of the suspension increased with an increase in micro-bead density. Because light travels from the bottom of the cuvette to the metal grid, scattering occurs before reaching the metal grid. Figure 3(b) displays the side view of the sample configuration containing scattering media. 


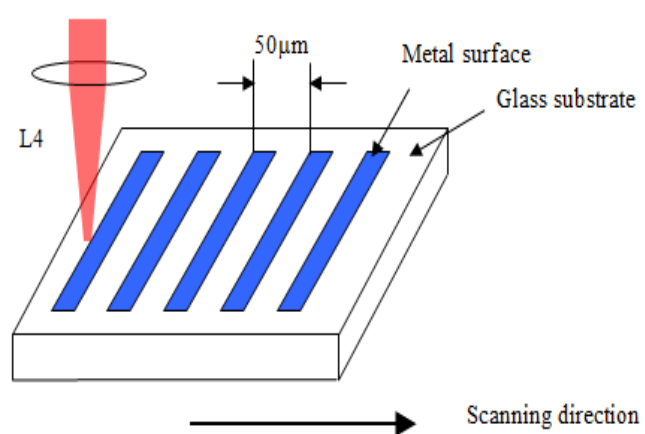

(a)

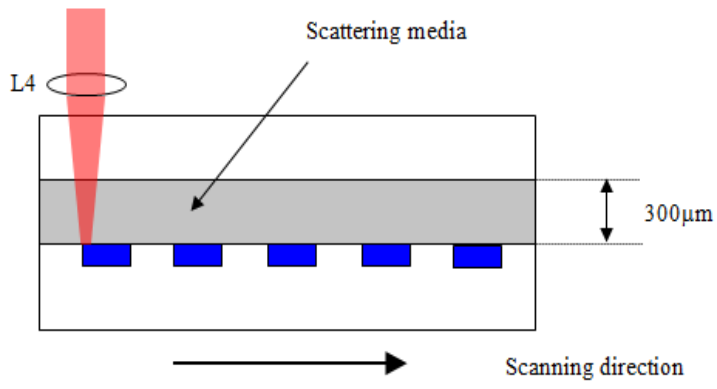

(b)

Fig. 3. (a) Configuration of a sample stage with a metal grid. (b) Side view of the sample stage with a metal grid covered with scattering media. Micro-beads ( $1 \mu \mathrm{m}$ diameter) in milk or deionized water are used as the scattering media.

A motorized stage holding the metal grid was synchronized with the image acquisition software. The stage was moved by $5 \mu \mathrm{m}$ and $30 \mathrm{~A}$-scans were recorded. The retro-reflector [RR] mounted on a voice coil was continuously driven by a function generator with a sinusoidal wave. The scan depth was adjusted to allow clear observation of the sample by controlling the peak-to-peak voltage of the sinusoidal wave at each sample. An actual scan depth is marked on each presented image. The sinusoidal signal was also connected to the input port of the acquisition board; acquisition software used the input port as an analog trigger signal. The trigger level was set to record an A-scan during the linear region of the sinusoidal signal. Once the program acknowledged a trigger event, a fixed number of data points were acquired from the detector output. After incoherent demodulation, the program displayed the recorded image on a computer screen. The acquisition program allows the user to predetermine the number of data points to acquire, sampling rate, low/high pass filter cutoff frequencies, and the lateral scanning step size (in micrometers). The acquisition program also allows data storage in a text-formatted file.

\section{Results}

A scattering media consisting of a suspension of micro-beads was placed above the metal lines. Figure 4(a) shows the OCT image of metal lines covered by a suspension of micro-beads without correction by adaptive optics. The suspension consisted of $1 \mu \mathrm{m}$ polystyrene microspheres diluted in deionized water. The thickness of the suspension chamber was $300 \mu \mathrm{m}$. The first bright line descending from the top of the image represents the boundary between the cover glass and the suspension chamber. The bright line at the bottom of the image represents blurred metal lines that ideally show as three distinct segments.

Figure 4(b) shows an OCT image of the metal lines through the suspension of micro-beads after correction with adaptive optics. The image displays three large segments, demonstrating that the beam diameter of the light is much smaller than the resolution of the lines.

The density of the micro-beads in the suspension was increased to create a stronger scattering media. Figure 5(a) displays an OCT image of the metal lines through a denser suspension of micro-beads. The upper bright line represents the boundary between the cover glass and the suspension. As micro-bead density increased, the brightness and thickness of the boundary increased simultaneously, potentially due to the increased difference in the refractive index between the two media or because microspheres attached to the glass surface were observed during the experiment. 
S1470 M.F. Shirazi et al. / Lateral resolution enhancement using programmable phase modulator in optical coherence tomography

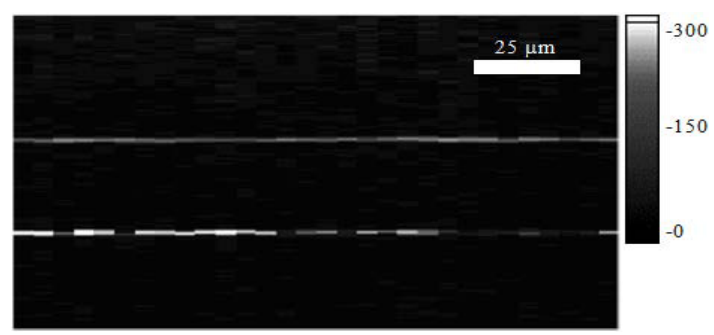

(a)

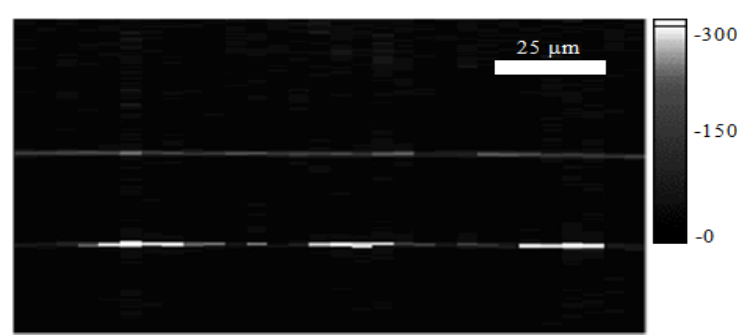

(b)

Fig. 4. (a) OCT image of metal lines through micro-bead suspension without aberration correction; (b) OCT image of metal lines through micro-bead suspension with aberration correction.

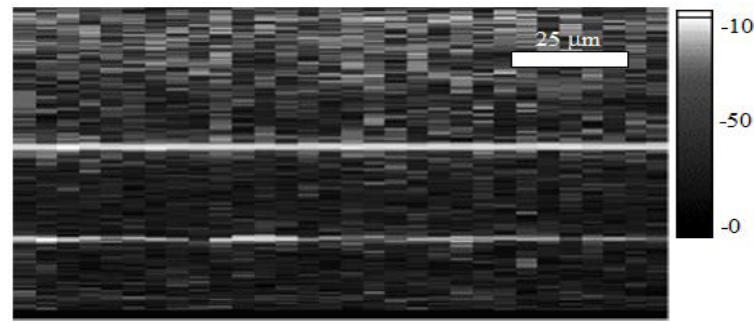

(a)

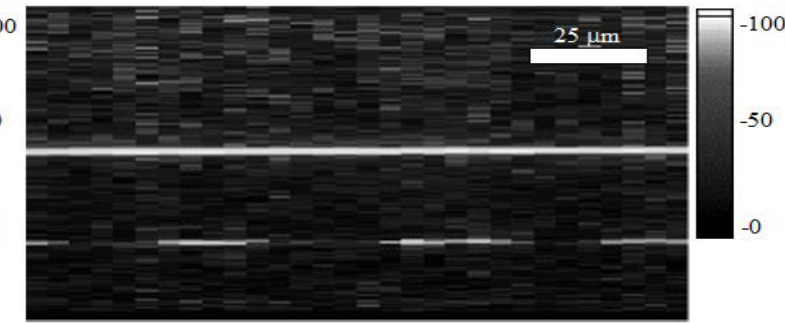

(b)

Fig. 5. (a) OCT image of metal grid through denser suspension of micro-beads without aberration correction; (b) OCT image of metal lines through denser suspension of micro-beads with aberration correction.

Figure 5(b) displays the OCT image of the metal lines with the adaptive optics control on, and demonstrates three distinct segments in the bottom line indicating that the aberration was successfully corrected.

\section{Conclusion}

The wavefront of the light entering the pupil of the sample path was measured using OCT. This approach has a few advantages over conventional wavefront sensors, including the use of an aberrometer and Shack-Hartmann wavefront sensors. The major benefits of using this method are an increased simplicity and a reduced cost. Because OCT is based on an interferometer, phase information can easily be obtained by analyzing a recorded interferogram and does not require additional optics or instruments. Another advantage is that the optical paths used to detect phase maps are same as the actual OCT imaging path. Therefore, no additional errors are introduced due to the use of different optics. A scattering sample was prepared with the metal lines obscured by a micro-bead suspension. In this case the aberration was successfully corrected by the adaptive optics, so the OCT image displayed clearer line separation in the sample as compare to without the adaptive optics.

\section{Acknowledgments}

This study was supported by a grant of the Korea Healthcare technology R\&D projects, Ministry for Health, Welfare \& Family Affairs, the Republic of Korea (A102024), Ministry of Education (MOE) and National Research Foundation of Korea (NRF) through the human resource training project for regional innovation (No. 2011-05-대-02-026) and also by a grant of the National Institute of Health 
(No. 201225940000), also this research was financially supported by the "Over regional linked 3D convergence industry promotion program" through the Ministry of Trade, Industry \& Energy (MOTIE) and Korea Institute for Advancement of Technology (KIAT) (N0. R0001512) and also this study was financially supported by Ministry of Health \& Welfare, Republic of Korea through the Korea Healthcare technology R\&D project (A102024-1011-0000200).

\section{References}

[1] M. Lombardo and G. Lombardo, Wave aberration of human eyes and new descriptors of image optical quality and visual performance, Journal of Cataract \& Refractive Surgery 36 (2010), 313-331.

[2] J. Liang and D.R. Williams, Aberrations and retinal image quality of the normal human eye, Journal of the Optical Society of America A 14 (1997), 2873-2883.

[3] U. Schmidt-Erfurth, R.A. Leitgeb, S. Michels, B. Považay, S. Sacu, B. Hermann, et al., Three-dimensional ultrahighresolution optical coherence tomography of macular diseases, Investigative Ophthalmology \& Visual Science 46 (2005), 3393-3402.

[4] G. Wollstein, L.A. Paunescu, T.H. Ko, J.G. Fujimoto, A. Kowalevicz, I. Hartl, et al., Ultrahigh-resolution optical coherence tomography in glaucoma, Ophthalmology 112 (2005), 229-237.

[5] Y. Ye, H. Jiang, M. Shen, B.L. Lam, D.C. Debuc, L. Ge, et al., Retinal oximetry using ultrahigh-resolution optical coherence tomography 6 (2012), 2085-2092.

[6] J.-Y. Lee, J. Ahn, T.W. Kim and B.S. Jeon, Optical coherence tomography in parkinson's disease: Is the retina a biomarker? Journal of Parkinson's Disease 4 (2014), 197-204.

[7] Y. Zhang, J. Rha, R. Jonnal and D. Miller, Adaptive optics parallel spectral domain optical coherence tomography for imaging the living retina, Optics Express 13 (2005), 4792-4811.

[8] M.J. Booth, Adaptive optical microscopy: The ongoing quest for a perfect image, Light Science \& Applications 3 (2014), e165.

[9] B.R. Patton, D. Burke and M.J. Booth, Adaptive Optics from Microscopy to Nanoscopy, Proceeding SPIE 8948, Multiphoton Microscopy in the Biomedical Sciences XIV, 894802 (2014).

[10] J.M. Bardsley, Wavefront reconstruction methods for adaptive optics systems on ground-based telescopes, SIAM Journal on Matrix Analysis and Applications 30 (2008), 67-83.

[11] J.W. Hardy, Active optics: A new technology for the control of light, Proceedings of the IEEE 66 (1978), 651-697.

[12] R. Tyson, Principles of Adaptive Optics, 3rd Edi., CRC Press, Taylor \& Francis Group, 2010.

[13] D.R. Williams, Imaging single cells in the living retina, Vision Research 51 (2011), 1379-1396.

[14] I. Kozak, Retinal imaging using adaptive optics technology, Saudi Journal of Ophthalmology 28 (2014), 117-122.

[15] A. Roorda, Adaptive optics for studying visual function: A comprehensive review, Journal of Vision 11 (2011), 1-21.

[16] M. Shirazi, W. Jung and J. Kim, Phase correction using programmable phase modulator (PPM) in optical coherence tomography, Biomedical Engineering Letters 4 (2014), 64-72. 